\title{
EXPRESSION OF THE SPIKE PROTEIN OF MURINE CORONAVIRUS JHM USING A
}

\section{BACULOVIRUS VECTOR}

\author{
Fumihiro Taguchi ${ }^{1}$ Sayaka Yoden ${ }^{1}$, Stuart Siddell ${ }^{2}$, and Tateki Kikuchi ${ }^{1}$ \\ National Institute of Neuroscience, NCNP, 4-1-1 \\ Ogawahigashi, Kodaira, Tokyo 187, Japan ${ }^{1}$ \\ Institute of Virology, University of Würzburg, Versbacher strasse 7 \\ 8700 Würzburg, Federal Republic of Germany²
}

\section{INTRODUCTION}

Coronaviruses are enveloped RNA viruses with single positive stranded genomic RNA of approximately 32 kilobases which encodes at least 3 structural proteins; a nucleocapsid protein $(\mathrm{N})$, a matrix glycoprotein $(\mathrm{M})$ and a spike glycoprotein $(\mathrm{S})$, as well as several nonstructural proteins (1). The $S$ protein forms the projecting spikes or peplomers on the surface of the virus particle (2). This protein is considered to be involved in the attachment of virus to susceptible cells, the induction of cell-to-cell fusion, and the induction of neutralizing antibodies $(3,4)$. It is also believed that the $S$ protein is important for determining the pathogenic potential of murine coronaviruses $(5,6)$. For more detailed structural and functional analyses of $S$ protein, we need to have large quantities of $S$ protein uncontaminated with other viral proteins. For this purpose, we have sought to express the $S$ gene product in a baculovirus expression vector. The Autographa californica nuclear polyhedrosis virus (AcNPV) insect baculovirus vector was chosen because of the high levels of foreign gene expression obtained using the AcNPV polyhedrin promoter (7). Also, translational modifications such as glycosylation, phosphorylation, and cleavage of the signal peptide are known to occur in this system. In this paper, we describe the expression of the MHV strain JHM (JHMV) S protein by recombinant baculovirus in insect cells, characterization of the protein and analysis of epitopes existing on the $\mathrm{S}$ protein.

\section{RESULTS}

\section{Isolation of a recombinant baculovirus containing the JHMV S gene}

To construct the baculovirus transfer vector containing the S gene of JHMV, the cDNA prepared from mRNA3 of JHMV (8) was inserted into the Bam H1 site of the transfer vector pAcYM1 (pAcYM1-S) in the correct orientation. Sequence analysis of the transfer vector has shown that the AUG codon of the S gene was located to be 75 bases downstream of the linker region. In order to obtain recombinant baculovirus containing the $S$ gene, pAcYM1-S DNA and infectious AcNPV DNA were co-transfected into insect Sf cells as described previously (9). Plaques produced by the progeny viruses from the transfection were screened for a polyhedrinnegative phenotype as well as $\mathrm{S}$ protein production in Sf cells by immunofluorescence. Among 4 polyhedrin-negative clones, 3 were shown to be recombinant viruses, since they produced $S$ protein in Sf cells.

Expression of the $S$ protein by recombinant baculovirus in insect cells and characterization of the polypeptide

Firstly the time course of synthesis of $S$ protein by the recombinant baculovirus was examined by ELISA. Extracts of Sf cells infected with the recombinant baculovirus 1 to 4 days 
before were adsorbed to microplates, and then the amount of expressed $S$ protein was determined by anti-JHMV serum. As a control, wild-type AcNPV-infected cell extracts were employed. The results showed that immunoreactive $S$ protein could be detected as early as two days post-infection and that the amount of $S$ protein was maximal at three days post-infection. Radiolabelled S protein expressed in insect cells was initially detected at 24 hours postinfection, and its synthesis was still detectable at 72 hours post-infection.

The size of the protein expressed by recombinant baculovirus was determined in a pulse-label experiment (Fig. 1A). Sf cells infected with baculoviruses or mock-infected cells were labelled with ${ }^{3} \mathrm{H}$-leucine for $30 \mathrm{~min}$ at 24 hours post-infection. Cell lysates were electrophoresed in SDS-polyacrylamide gels. As shown in Fig. 1A, the recombinant virus synthesized a protein that migrated with an estimated molecular weight of $150 \mathrm{~K}$, similar to that of the co-translationally glycosylated JHMV S protein synthesized in infected DBT cells. A protein of this molecular weight was not observed in cells infected with wild-type AcNPV or mock-infected Sf cells. The $150 \mathrm{~K}$ protein was examined by immune precipitation with polyclonal anti-JHMV mouse serum. As shown in Fig. 1B, the $150 \mathrm{~K}$ protein was precipitated from cells infected with the recombinant virus by anti-JHMV antibodies. As expected, the antiJHMV serum also precipitated the $60 \mathrm{~K}$ mol. wt. N protein synthesized in JHMV-infected cells (Fig. 1B). In wild-type AcNPV infected Sf cells, a strong band with a molecular weight of $33 \mathrm{~K}$ was detected, which has been shown to be non-specifically precipitated polyhedrin protein resulting from incomplete solubilization of the occlusions in the immunoprecipitation buffer (Fig. 1B). These data indicate that a protein indistinguishable in terms of electrophoretic pattern from $S$ protein produced in JHMV-infected DBT cells was produced by recombinant baculovirus in insect cells.

In order to determine whether carbohydrate chains were attached to $S$ protein produced in insect cells, Sf cells infected with recombinant virus were treated with various concentration of tunicamycin and immunoprecipitated with anti-JHMV serum. As shown in Fig. 2, the molecular weight of the $S$ protein expressed in the presence of $10 \mu \mathrm{g} / \mathrm{ml}$ of tunicamycin was reduced to approximately 130K, which was also observed in DBT cells infected with JHMV. These results indicated that glycosylation of S protein was carried out in insect cells after recombinant virus infection. To assess proteolytic cleavage of the $S$ protein expressed in insect cells, Sf cells infected with the recombinant virus were pulse-chase labelled with ${ }^{3} \mathrm{H}$-leucine and cell lysates were immunoprecipitated with anti-JHMV serum. Although cleavage of the $S$ protein produced in mouse cells infected by JHMV occurs within 180 minutes, no cleavage products were detected in the insect cells during a 4-hour chase. The localisation of the $S$ protein expressed in insect cells was studied by immunofluorescence. Sf cells were infected with the recombinant virus and 3 days later, cells were fixed with acetone. The fixed cells were incubated with anti-JHMV antibodies and stained with FITC-conjugated anti-mouse IgG. The $S$ protein expressed in insect cells could be localized on the cell surface. No JHMV-related antigen was detected within the AcNPV-infected cells or mock-infected cells. The surface location of the $S$ protein found in cells infected with the recombinant virus was confirmed by immunofluorescence using unfixed cells.

\section{Production and characterisation of antisera to expressed $S$ protein in rats}

To see whether the $S$ protein produced by the recombinant baculovirus elicited antibodies, rats and mice were immunized with sucrose gradient-purified material. A lysate of Sf cells infected with recombinant baculovirus was loaded onto a 10 to $50 \%$ (w/w) sucrose gradient and centrifuged. The fractions with high ELISA titres were pooled; the pool was shown to have an ELISA titre of 1:625 and a protein concentration of $3 \mathrm{mg} / \mathrm{ml}$. Sera collected from the animals immunized with this material were serologically tested. Rats immunized with the $S$ protein produced antibodies which reacted by immunoprecipitation with the $S$ protein synthesized in DBT cells infected with JHMV (Fig. 3). In immunofluorescence DBT cells infected with JHMV were stained by the antisera. However in the plaque reduction assay the antisera failed to neutralize the infectivity of JHMV (neutralization titre <1:5). Since mice immunized with partially purified $S$ protein did not produce detectable levels of antibodies against $S$ protein in immunofluorescence or immunoprecipitation, alternative immunizations were carried out, i.e., immunization with the intact $S$ fells carrying expressed $S$ protein or the lysate of Sf cells infected with the recombinant baculovirus. However, none of these immunizations stimulated detectable antibody production in mice. 


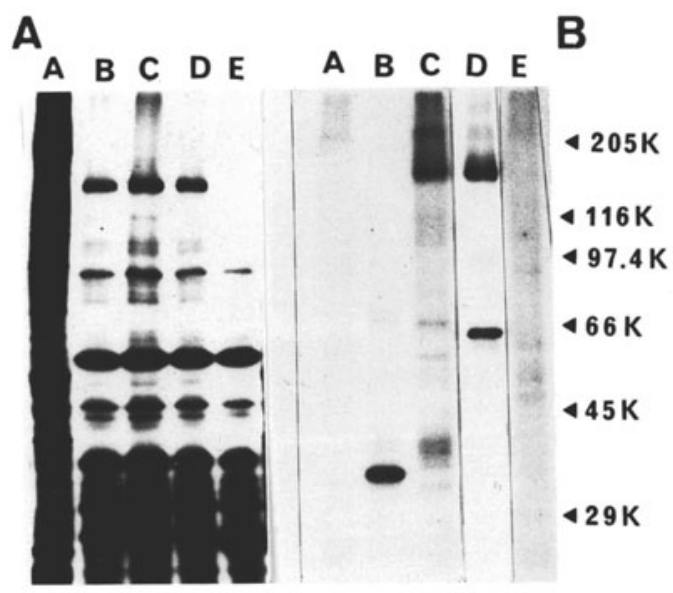

Fig. 1. Expression of $\mathrm{S}$ protein in Sf cells by recombinant baculovirus. A. The protein synthesized in Sf cells infected with three recombinant baculoviruses (B, C, D), Sf cells infected with wild-type baculovirus (E), or mock-infected Sf cells (A) were labelled with ${ }^{3} \mathrm{H}$ leucine. Cell lysates were analysed by $10 \%$ SDS-PAGE. B. Immunoprecipitation with antiJHMV serum of ${ }^{3} \mathrm{H}$-leucine-labelled proteins from $\mathrm{Sf}$ cells infected with recombinant baculovirus (C), Sf cells infected with wild-type baculovirus (B), mock-infected Sf cells (A), JHMV-infected DBT cells (D) and mock-infected DBT cells (E). Immune precipitates were analysed by $10 \%$ SDS-PAGE.
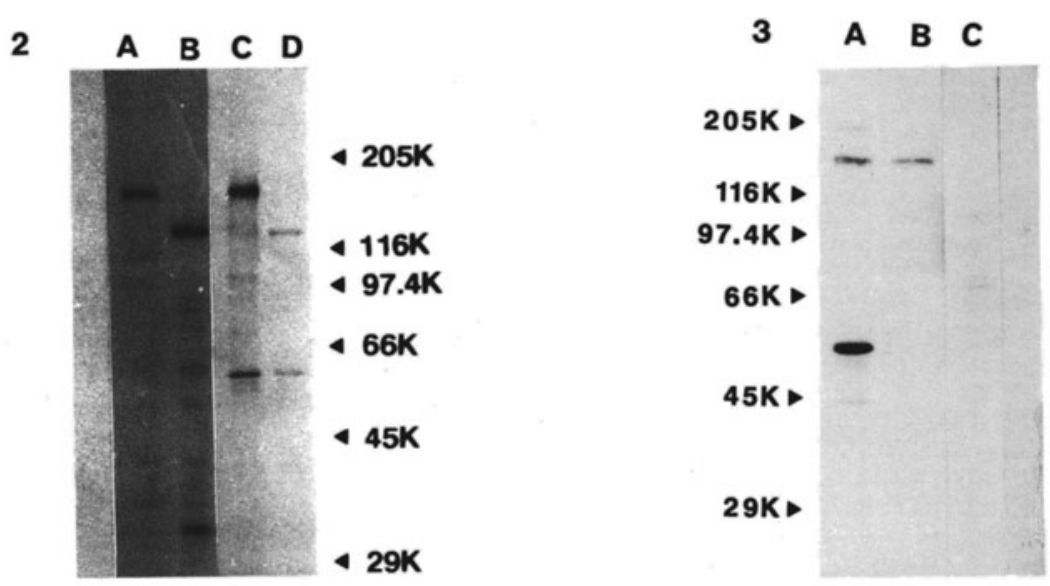

Fig. 2. Effects of tunicamycin (TM) on the expression of $\mathrm{S}$ protein in $\mathrm{Sf}$ cells by recombinant baculovirus. Sf cells infected with recombinants were treated with 0 (A) or $10 \mu \mathrm{g} / \mathrm{ml}$ (B) of TM and cell lysates were immunoprecipitated with anti-JHMV serum. As a control, JHMV-infected DBT cells treated with $0(\mathrm{C})$ or $4 \mu \mathrm{g} / \mathrm{ml}$ of TM were similarly immunoprecipitated. Precipitates were analysed by $10 \%$ SDS-PAGE.

Fig. 3. Detection of anti-S antibodies in rat serum immunized with $S$ protein expressed in $S f$ cells by a recombinant baculovirus. The lysates from JHMV-infected (A, B) and mock-infected DBT cells $(C)$ were immunoprecipitated with the serum of immunized rat $(B, C)$ and with antiJHMV mouse serum (A). Immune precipitates were analysed by $10 \%$ SDS-PAGE. 
Table 1

Reactivity of monoclonal antibodies to S1 and S2 proteins expressed by recombinant baculoviruses $^{1}$

\begin{tabular}{ccccccc} 
& \multicolumn{3}{c}{$\mathrm{NT}^{2}$ on } & \multicolumn{4}{c}{ Immunofluorescence against } \\
& \multicolumn{2}{c}{ virus isolates } & \multicolumn{3}{c}{ proteins expressed by baculovector } \\
Monoclonal Ab & $\mathrm{cl-2}$ & $\mathrm{sp4}$ & $\mathrm{S}$ & $\mathrm{S} 1$ & $\mathrm{~S} 2$ & Mock $^{3}$ \\
\hline $32-4$ & + & + & + & + & - & - \\
$110-3$ & + & + & + & + & - & - \\
$75-4$ & + & + & + & + & - & - \\
$8-1$ & + & + & + & + & - & - \\
$141-3$ & - & - & + & + & - & - \\
$104-2$ & - & - & + & + & - & - \\
$29-6$ & - & - & - & - & + & - \\
$80-1$ & + & - & - & - & - & -
\end{tabular}

1. All monoclonal antibodies were shown to recognize $S$ protein of $\mathrm{cl}-2$ by immunofluorescence.

2. Neutralization test.

3. Sf cells infected with wild type baculovirus.

\section{Expression of S1 and S2 proteins by recombinant baculoviruses.}

Since we could not detect cleavage products of $S$ protein in insect cells, we cut the cDNA with Cla I, which gives 2 cDNA fragments. The fragment from the 5 ' half encodes a protein which is 13 amino acids larger than the deduced cleavage product of JHMV and is designated S1. The other fragment derived from the 3' half encodes a polypeptide 12 amino acids shorter than the other deduced cleavage product and was named S2. Recombinant baculoviruses containing these fragments were isolated and the properties of S1 and S2 expressed in insect cells by the recombinants were examined. Using immune precipitation, S1 and S2 proteins with molecular weights of $95 \mathrm{~K}$ and $63 \mathrm{~K}$, respectively, were shown to be produced in insect cells by the recombinants. S1 was shown to be glycosylated using tunicamycin. However, S2 was not glycosylated probably because the protein lacks a signal peptide. These two proteins were used in an attempt to elicit antibodies in rats. High titred antibodies from rats against $\mathrm{S} 2$ were detected by immunofluorescence and immunoprecipitation. However, no antibodies recognizing S1 were detected. Since these 2 proteins were well precipitated by anti-JHMV mouse serum, it seems likely that these proteins retain the antigenicity of S protein. We therefore used these proteins to study the localization of epitopes recognized by the monoclonal antibodies (McAbs) produced by immunizing with cl-2 virus which contains an $S$ protein larger than that encoded by the cDNA clone used in these experiments (10). As shown in Table 1, among $8 \mathrm{McAbs}$ examined, 80-1 could not react with $\mathrm{S}, \mathrm{S} 1$ or $\mathrm{S} 2$ and was probably specific to the large S protein of cl-2 as reported previously (11). Of the 7 McAbs, 6 reacted to S1 as well as S by immunofluorescence. All McAbs with neutralizing activity to sp-4 reacted with S1 but not S2. These data suggest that the majority of epitopes which elicit neutralizing antibodies are located in the N-terminal half of S protein.

\section{DISCUSSION}

In the present paper, we have demonstrated high level expression of the JHMV S protein in insect cells infected with recombinant baculoviruses. The $S$ protein expressed by recombinant baculovirus in insect cells was shown to correspond in size and antigenicity to the glycosylated $S$ protein produced in mouse cells infected with JHMV and to be expressed on the cell surface as was observed in JHMV-infected mouse cells. However, the post-translational processing of the $S$ protein expressed in insect cells was shown to be different from that in JHMV-infected mouse cells. For example, proteolytic cleavage, which is believed to be a prerequisite for the fusogenic activity of the JHMV S protein was not observed. Also further studies will be necessary to determine if the complex post-translational processing of the JHMV $\mathrm{S}$ carbohydrate components is identical in insect and mouse cells. 
Rats immunized with the $S$ protein expressed in insect cells produced antibodies against the $S$ protein. In previous reports, antibodies raised against viral surface glycoproteins expressed in the baculovirus system have been shown to neutralize infectivity of the virus (12). Unexpectedly, the antibodies which we obtained failed to neutralize the infectivity of JHMV, although these antibodies bound to the $S$ protein produced in mouse cells. There are several possible explanations. Firstly, as suggested above, there might be differences in the carbohydrate structure between the $S$ protein expressed in insect cells and that produced in mouse cells. Such differences may influence epitopes involved in eliciting neutralizing antibodies. Secondly the uncleaved form of the $S$ glycoprotein might not be able to induce neutralizing antibodies in animals. Thirdly, it is possible that the S cDNA clone used (or the RNA molecule from which it was produced) acquired mutations critical to the epitopes responsible for eliciting neutralizing antibodies. We consider this possibility unlikely, because of 6 different monoclonal antibodies directed against the $S$ protein, four of which are neutralizing antibodies, all reacted well with the $S$ protein expressed in insect cells. The last possibility is that the antibodies obtained after immunization with $S$ protein were mostly against epitopes residing in the $C$ terminal half of the $S$ protein. This is supported by the observtion that the immunogenicity of $S 1$, the $\mathrm{N}$ terminal half of the $\mathrm{S}$ protein, was shown to be very weak, while that of S2 was fairly strong as shown by the cDNA fragment experiments, and that with monoclonal antibody analysis, the majority of neutralising epitopes were suggested to reside in $S 1$. In our view many of these questions can be further investigated by expressing the $\mathrm{S}$ glycoprotein in mouse cells using the vaccinia virus system.

\section{ABSTRACT}

The spike (S) protein of murine coronavirus JHM strain (JHMV) has been expressed in insect cells using a recombinant baculovirus vector. The expressed $S$ protein was shown to be glycosylated and expressed on the cell surface, and to be similar in size and antigenic properties to the $S$ protein produced in mouse cells infected by JHMV. However, no proteolytic cleavage was detected in insect cells. The sera from rats immunised with $S$ protein derived from insect cells reacted in immunoprecipitation and immunofluorescence with the $S$ protein produced in JHMV-infected mouse cells. However, the antisera failed to neutralize the infectivity of JHMV. The studies on two proteins expressed by recombinant baculoviruses, corresponding to the cleavage products $S 1$ and S2, and a panel of monoclonal antibodies suggest that the majority of epitopes which elicit the neutralizing antibodies are present in the $\mathrm{N}$ terminal half of the $\mathrm{S}$ protein.

\section{ACKNOWLEDGEMENTS}

The authors thank Mrs. H. Hirano for technical assistance. This work was partly supported by the Grant-in-aid from Ministry of Health and Welfare of Japan.

\section{REFERENCES}

1. Siddell, S., Wege, H. and ter Meulen, V. (1983) The biology of coronaviruses. J. gen. Virol. 64, 761-776.

2. Sturman, L.S. and Holmes, K.V. (1983) The molecular biology of coronaviruses. Adv. Virus Res. 18, 35-112.

3. Holmes, K.V., Doller, E.W. and Behnke, J.N. (1981) Analysis of the function of coronavirus glycoprotein by differential inhibition of synthesis with tunicamycin. Adv. Exp. Med. Biol. 142, 133-142.

4. Collins, A.R., Knobler, R.L., Powell, H. and Buchmeier, M. (1982) Monoclonal antibodies to murine hepatitis virus-4 (strain JHM) define the viral glycoprotein responsible for attachment and cell-cell fusion. Virology 119, 358-371.

5. Fleming, J.O., Trousdale, M.D., El-Zaatari, F.A.K., Stohlman, S.A. and Weiner, L.P. (1986) Pathogenicity of antigenic variants of murine coronavirus JHM selected with monoclonal antibodies. J. Virol. 58, 869-875.

6. Wege, H., Winter, J. and Meyermann, R. (1988) The peplomer protein E2 of coronavirus JHM as a determinant of neurovirulence: Definition of critical epitopes by variant analysis. J. gen. Virol. 69, 87-98.

7. Luckow, V.A. and Summers, M.D. (1988) Trends in the development of baculovirus expression vectors. Bio/Technology $6,47-55$. 
8. Schmidt, I., Skinner, M. and Siddell, S. (1987) Nucleotide sequence of the gene encoding the surface projection glycoprotein of coronavirus JHV-JHM. J. gen. Virol. 68, 47-56.

9. Matsuura, Y., Possee, R.D., Overton, H. and Bishop, D.H. (1986) Expression of the Scoded genes of lymphocytic choriomeningitis arenavirus using baculovirus vector. J. gen. Virol. 67, 1515-1529.

10. Taguchi, F., Siddell, S., Wege, H. and ter Meulen, V. (1985) Characterisation of a variant virus selected in rat brains after infection by coronavirus mouse hepatitis virus JHM. L. Virol. 54, 429-435.

11. Taguchi, F. and Fleming, J.O. (1989) Comparison of six different murine coronavirus JHM variants by monoclonal antibodies against the E2 glycoprotein. Virology, 169, 233-235.

12. Kuorda, K., Hauser, C., Rudolf, R., Klenk, H.-D and Doerfler, W. (1986) Expression of the influenza virus haemagglutinin in insect cells by a baculovirus vector. EMBO J. 5 , 1359-1365. 